\title{
Correcting Fast-Mode Pressure Errors in Storm-Scale Ensemble Kalman Filter Analyses
}

\author{
Corey K. Potvin ${ }^{1,2}$ and Louis J. Wicker ${ }^{2}$ \\ ${ }^{1}$ Cooperative Institute for Mesoscale Meteorological Studies, University of Oklahoma, USA \\ ${ }^{2}$ NOAA/OAR/National Severe Storms Laboratory, National Weather Center, \\ 120 David L. Boren Boulevard, Norman, OK 73072, USA
}

Correspondence should be addressed to Corey K. Potvin; corey.potvin@noaa.gov

Received 29 May 2013; Revised 26 July 2013; Accepted 19 August 2013

Academic Editor: Ming Xue

Copyright (C) 2013 C. K. Potvin and L. J. Wicker. This is an open access article distributed under the Creative Commons Attribution License, which permits unrestricted use, distribution, and reproduction in any medium, provided the original work is properly cited.

\begin{abstract}
A typical storm-scale ensemble Kalman filter (EnKF) analysis/forecast system is shown to introduce imbalances into the ensemble posteriors that generate acoustic waves in subsequent integrations. When the EnKF is used to research storm-scale dynamics, the resulting spurious pressure oscillations are large enough to impact investigation of processes driven by nonhydrostatic pressure gradient forces. Fortunately, thermodynamic retrieval techniques traditionally applied to dual-Doppler wind analyses can be adapted to diagnose the balanced portion of an EnKF pressure analysis, thereby eliminating the fast-mode pressure oscillations. The efficacy of this approach is demonstrated using a high-resolution supercell thunderstorm simulation as well as EnKF analyses of a simulated and a real supercell.
\end{abstract}

\section{Introduction}

The EnKF [1] has become a popular and valuable tool for storm-scale research [2-12]. Particularly when dual-Doppler radar data are available, EnKF data assimilation can provide reliable analyses of wind and, to a lesser degree, temperature and microphysical variables in convective storms. EnKF analyses of pressure, on the other hand, are subject to severe errors, at least with some compressible model configurations (the first tests of the EnKF with a compressible model were performed by Tong and Xue [5]). This problem is illustrated in Figure 1 using output from the National Severe Storms Laboratory Collaborative Model for Multiscale Atmospheric Simulation (NCOMMAS; $[13,14]$ ) ensemble square root filter [15]. Similar behavior occurs using the Data Assimilation Research Testbed [16] EnKF with the Advanced Research Weather Research and Forecasting (WRF-ARW; [17]) model (James Marquis and Thomas Jones, personal communication 2013). The pressure analysis errors severely impede investigation of critical storm processes that are, in part, driven by dynamic pressure gradient forces, including supercell occlusion downdrafts [18], lifting of negatively buoyant air
[19], supercell propagation [20], the descending rear inflow and ascending front-to-rear flow in mesoscale convective systems [21], and possibly descending reflectivity cores [22].

The pressure oscillations shown in Figure 1 are associated with acoustic waves generated within the data assimilation region. The waves are presumably excited as each ensemble member adjusts to an updated initial condition that is dynamically inconsistent with the model (i.e., unbalanced). This hypothesis is supported by two observations. First, the acoustic waves occur whether or not pressure is updated during the data assimilation and therefore cannot be attributed to erroneous ensemble covariances between the pressure and other variables (though the latter could conceivably exacerbate the problem in cases where pressure is updated). Second, spurious waves are not evident in perfect-model EnKF observing system simulation experiments (OSSEs) with the NCOMMAS (not shown), indicating that the waves arise only when analysis increments are substantially unbalanced. The generation of high-amplitude fast modes due to unbalanced initial conditions is a long-recognized problem in numerical weather prediction, and many approaches have been used to improve dynamical balance during the data assimilation 

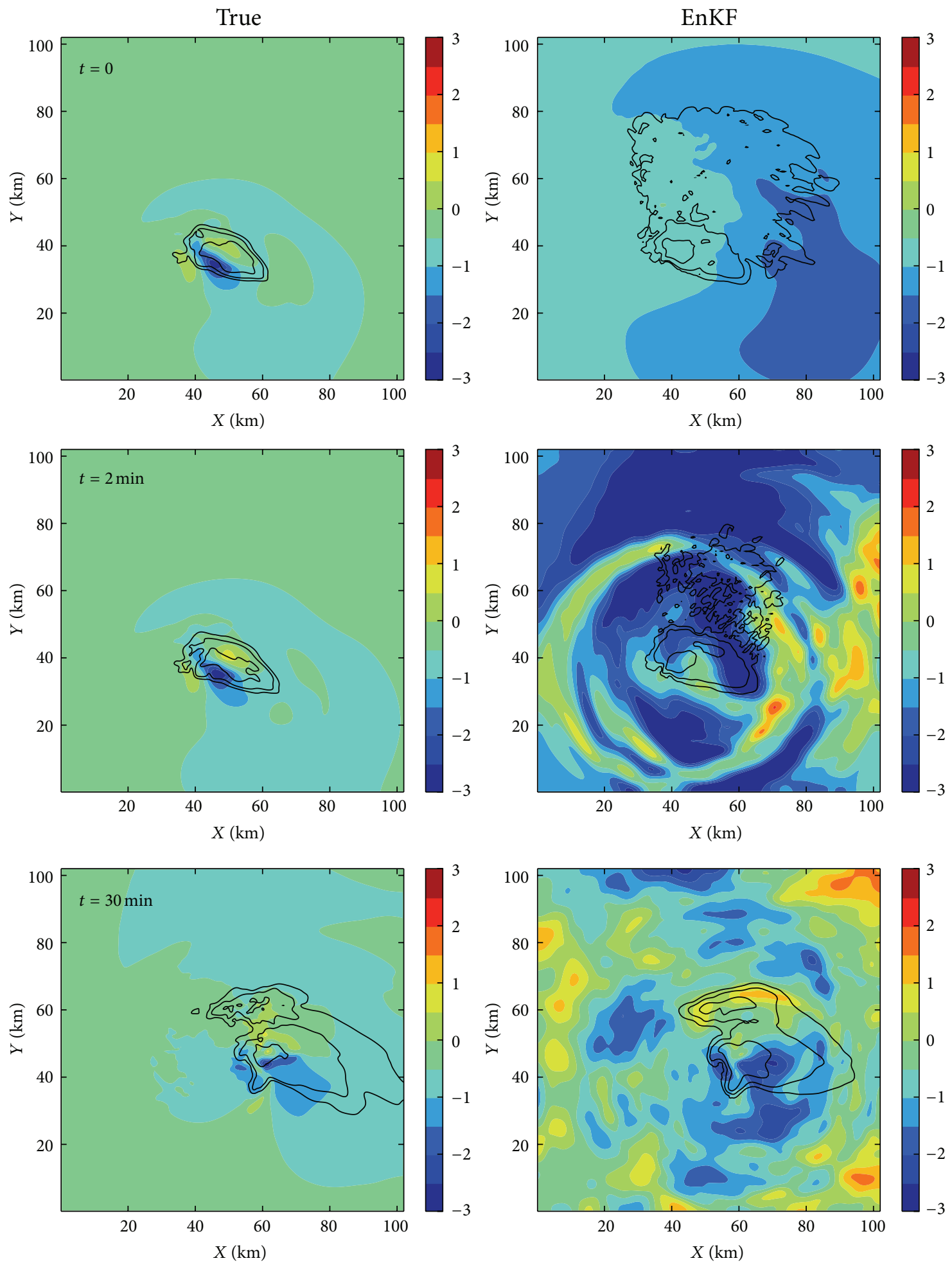

Figure 1: True (left column) and EnKF mean posterior (right column) $p^{\prime}$ (shading; hPa) and radar reflectivity factor (contoured at 10, 30, and $50 \mathrm{dBZ}$ ) at $z=0.9 \mathrm{~km}$. Fields are valid after (top row) zero, (middle row) one, and (bottom row) fifteen 2 min forecast cycles. The true $p^{\prime}$ were filtered and averaged as in Potvin et al. [23] to permit more direct comparisons with the (coarser) EnKF $p^{\prime}$. 

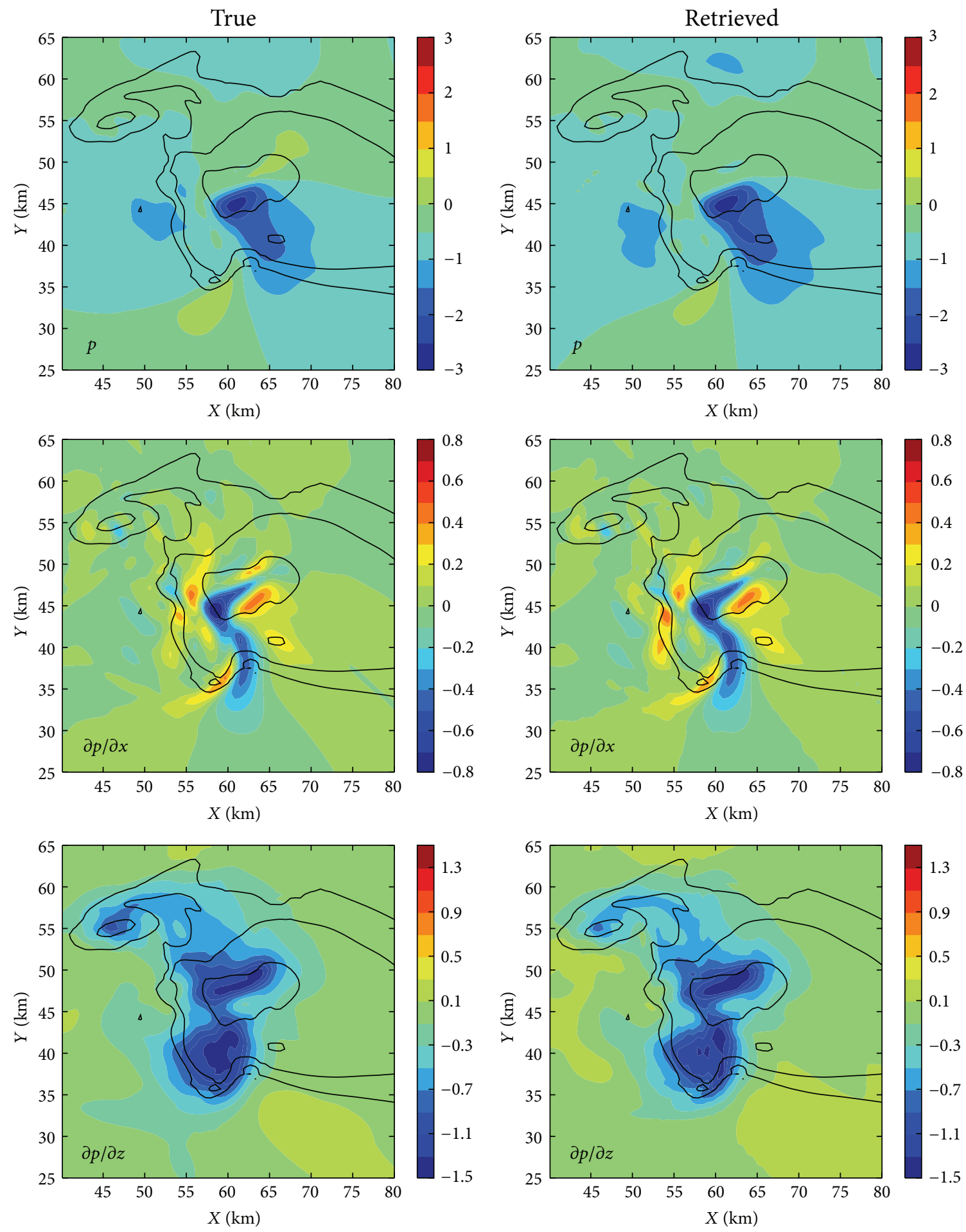

Figure 2: True (left) and retrieved (right) $p$ (top; hPa), $\partial p / \partial x$ (middle; hPa km ${ }^{-1}$ ), and $\partial p / \partial z\left(\right.$ bottom; hPa km $\left.{ }^{-1}\right)$ at $z=0.9 \mathrm{~km}$. Radar reflectivity factor is contoured at 10,30 , and $50 \mathrm{dBZ}$.

procedure [24, 25]. Given the small influence of the pressure field on the remaining state variables in certain compressible cloud models on the relevant spatiotemporal scales, errors due to the acoustic waves are largely confined to the pressure field in storm-scale EnKF analyses. This makes it possible to retrieve the portion of the pressure field that is in balance with the remaining model fields (hereafter, the "balanced" pressure). This obviates the need to mitigate the acoustic waves during the data assimilation procedure, at least when the analysis is not being used to initialize a numerical forecast. 

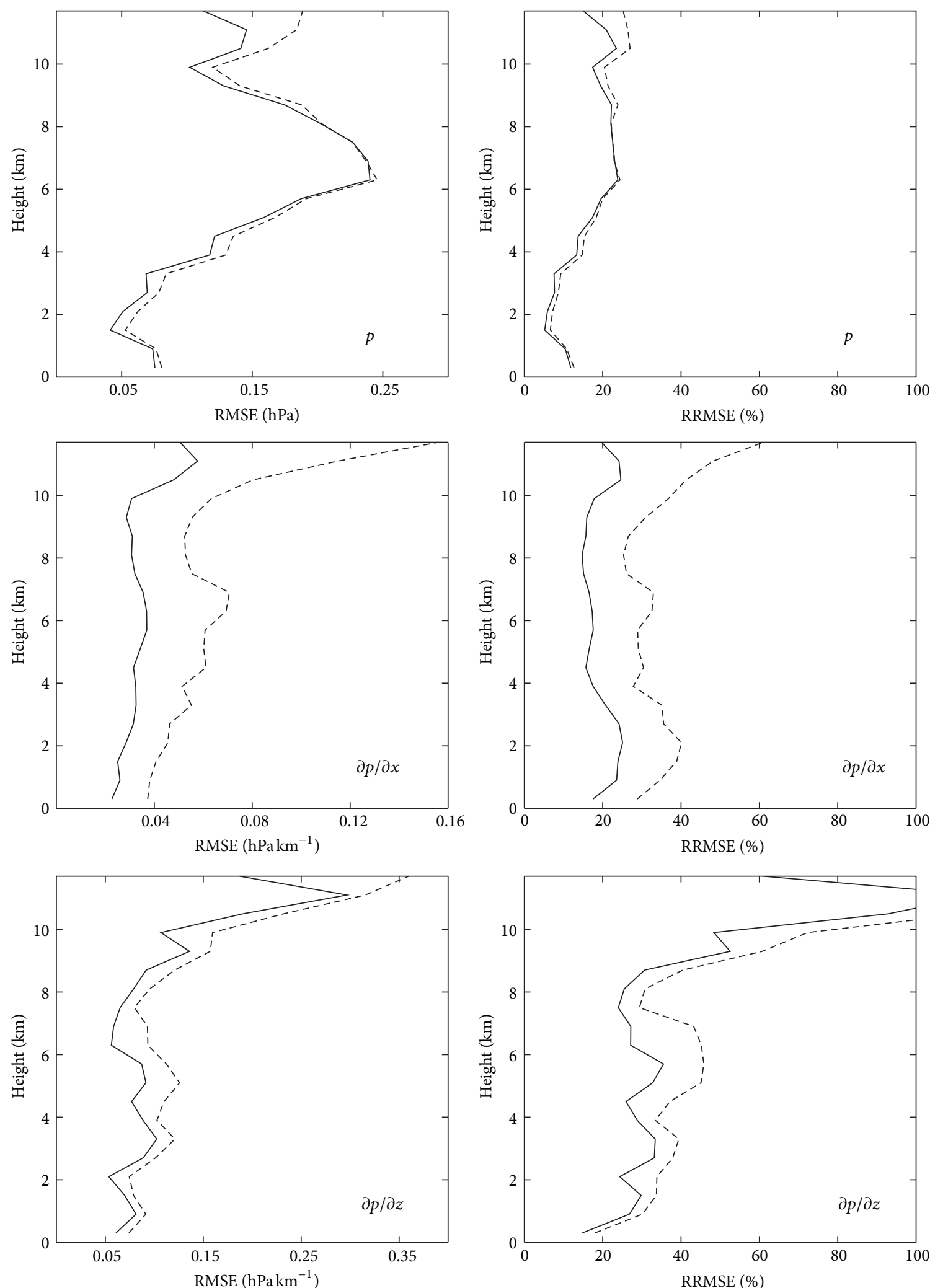

FIGURE 3: Vertical profiles of RMSE (left column) and RRMSE (right column) $p_{\text {final }}$ (top row), $\partial p_{\text {final }} / \partial x$ (middle row), and $\partial p_{\text {final }} / \partial z$ (bottom row) for default retrieval ( $\Delta t=30 \mathrm{~s}$; solid) and retrieval with $\Delta t=120 \mathrm{~s}$ (dashed). All quantities valid where the true $\mathrm{dBZ}>0$. 


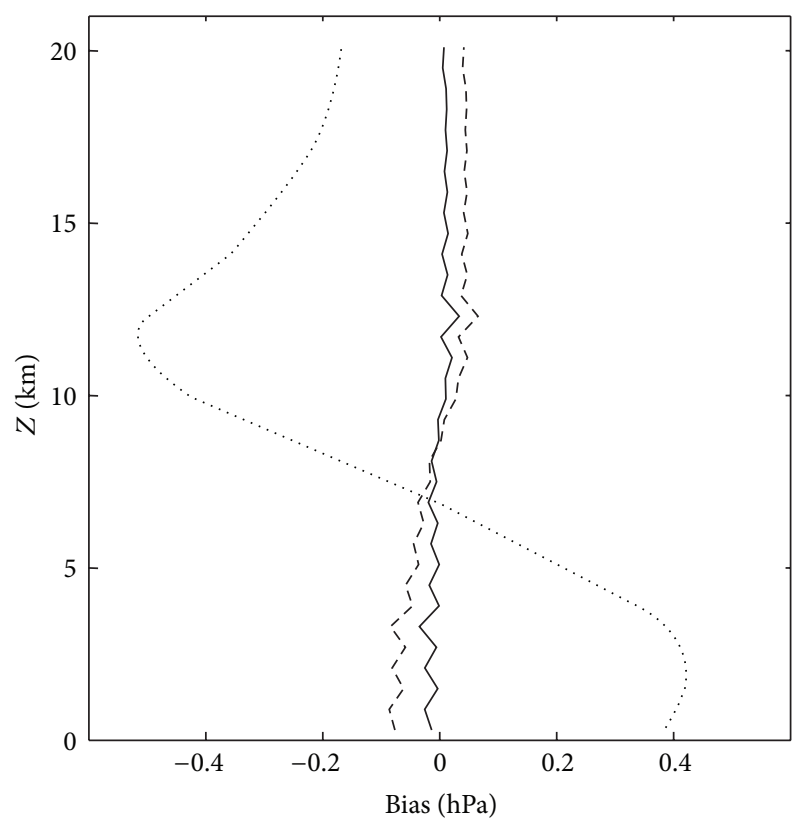

FIGURE 4: Vertical profiles of horizontal domain-wide bias in $p_{2 \mathrm{D}}$ (dotted), $p_{\text {final }}$ (solid), and $p_{\text {final }}$ retrieved with the hydrometeor drag term omitted in the cost function (dashed).

Instead, the balanced pressure can be retrieved after the data assimilation is complete.

Several methods are available for diagnosing perturbation (from the hydrostatic base state) pressure, typically in its nondimensional Exner function form $\pi$ (the prime symbol is omitted herein to simplify the notation), from the equations of motion. Early methods satisfied the horizontal equations of motion on individual horizontal planes [26, 27]. A major limitation of that approach is that the analyzed $\pi$ is offset from the true $\pi$ by a vertically varying constant, precluding unique solution of $\partial \pi / \partial z$ unless independent pressure measurements are available at each analysis level. This problem is avoided when all three equations of motion are satisfied [28-30], in which case the retrieved $\pi$ is offset by a volume-wide (rather than vertically varying) constant, thereby permitting the impact of vertical pressure gradients to be quantitatively considered. The tradeoff is that errors in the analyzed local derivative and buoyancy terms in the vertical momentum equation typically result in $\partial \pi / \partial x$ and $\partial \pi / \partial y$ analyses that are inferior to those obtained using twodimensional retrieval. As will be shown, however, there is a very simple procedure for obtaining the advantages of both the $2 \mathrm{D}$ and $3 \mathrm{D}$ approaches. This combined method permits useful pressure retrievals to be obtained from acoustic wavecontaminated EnKF analyses.

\section{Pressure Retrieval Method}

We adopt a variational framework for our pressure retrieval scheme. The momentum equation constraints use the Klemp and Wilhelmson [31] formulation of the equations of motion except with the Coriolis term (which is negligible) omitted.
A horizontal smoothness constraint is imposed to filter noise. The cost function we seek to minimize can therefore be expressed as

$$
J=\frac{1}{2} \sum_{i} \sum_{j} \sum_{k} C_{1} J_{1}^{2}+C_{2} J_{2}^{2}+C_{3} J_{3}^{2}+C_{4} J_{4}^{2}
$$

where

$$
\begin{array}{cc}
J_{1}=\left(\frac{\partial \pi}{\partial x}-F\right), & J_{2}=\left(\frac{\partial \pi}{\partial y}-G\right), \\
J_{3}=\left(\frac{\partial \pi}{\partial z}-g \frac{\theta^{\prime}}{\theta_{0}^{2}}-H\right), & J_{4}=\left(\frac{\partial^{2} \pi}{\partial x^{2}}+\frac{\partial^{2} \pi}{\partial y^{2}}\right) ;
\end{array}
$$

$i, j$, and $k$ are the model grid indices; $F, G$, and $H$ are the sums of the local time derivative, advection, and turbulent mixing terms for the $u$-, $v$-, and $w$-equations, respectively; $g$ is the gravitational acceleration; and $\theta_{0}$ and $\theta^{\prime}$ are the horizontally homogeneous base state and perturbation potential temperature, respectively. The subscripted $C$ 's represent the weighting coefficients for each respective constraint, computed similarly to the coefficients in [30]:

$$
\begin{aligned}
& C_{1}=\left[\frac{\sum_{i} \sum_{j} \sum_{k}\left(F^{2}\right) \Delta x \Delta y \Delta z}{\sum_{i} \sum_{j} \sum_{k} \Delta x \Delta y \Delta z}\right]^{-1}, \\
& C_{2}=\left[\frac{\sum_{i} \sum_{j} \sum_{k}\left(G^{2}\right) \Delta x \Delta y \Delta z}{\sum_{i} \sum_{j} \sum_{k} \Delta x \Delta y \Delta z}\right]^{-1}, \\
& C_{3}=\left[\frac{\sum_{i} \sum_{j} \sum_{k}\left(H^{2}\right) \Delta x \Delta y \Delta z}{\sum_{i} \sum_{j} \sum_{k} \Delta x \Delta y \Delta z}\right]^{-1}, \\
& C_{4}=k_{0}^{-4},
\end{aligned}
$$

where $\Delta x, \Delta y$, and $\Delta z$ are the analysis grid spacings and $k_{0}$ is the cutoff wavenumber, for which the theoretical filter response is 0.5 . The present study uses a $3 \Delta x$ cutoff wavelength.

In the first step of the pressure retrieval scheme, a $2 \mathrm{D}$ retrieval is performed (i.e., $C_{3}$ is set to zero) over each analysis level. The resulting analysis, $\pi_{2 \mathrm{D}}$, is stored, and then a $3 \mathrm{D}$ retrieval is performed, yielding $\pi_{3 \mathrm{D}}$. The vertically varying constant $A(k)$ by which $\pi_{2 \mathrm{D}}$ is offset from the balanced $\pi$, $\pi_{\text {bal }}$, is then estimated by computing the mean difference between $\pi_{3 \mathrm{D}}$ and $\pi_{2 \mathrm{D}}$ for each $k$. To see why $A(k)$ can be estimated in this way, consider that the $2 \mathrm{D}$ and $3 \mathrm{D}$ analyses can be written as

$$
\begin{gathered}
\pi_{2 \mathrm{D}}=\pi_{\text {bal }}+A(k)+\pi_{2 \mathrm{D}}^{\text {error }}, \\
\pi_{3 \mathrm{D}}=\pi_{\text {bal }}+\pi_{3 \mathrm{D}}^{\text {error }},
\end{gathered}
$$

where $\pi_{2 \mathrm{D}}^{\text {error }}$ and $\pi_{3 \mathrm{D}}^{\text {error }}$ are the retrieval errors, apart from the vertically varying constant. Assuming that the $2 \mathrm{D}$ and 3D retrievals are approximately unbiased relative to each other (apart from the vertically varying constant), averaging the differences between the retrievals over each horizontal analysis plane (symbolized by brackets) yields

$$
\left\langle\pi_{2 \mathrm{D}}-\pi_{3 \mathrm{D}}\right\rangle=\left\langle\pi_{2 \mathrm{D}}^{\text {error }}-\pi_{3 \mathrm{D}}^{\text {error }}\right\rangle+A(k) \approx A(k) .
$$




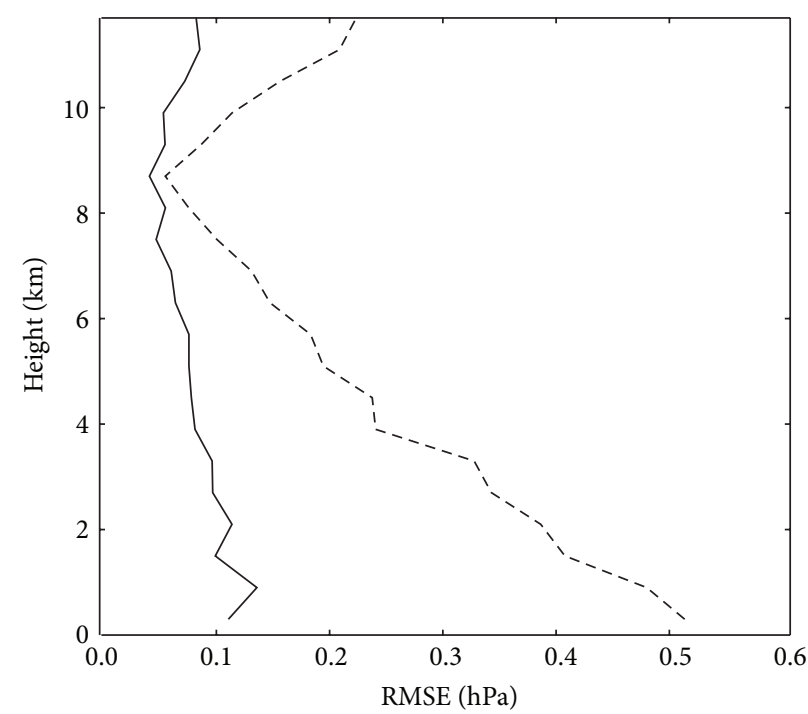

(a)

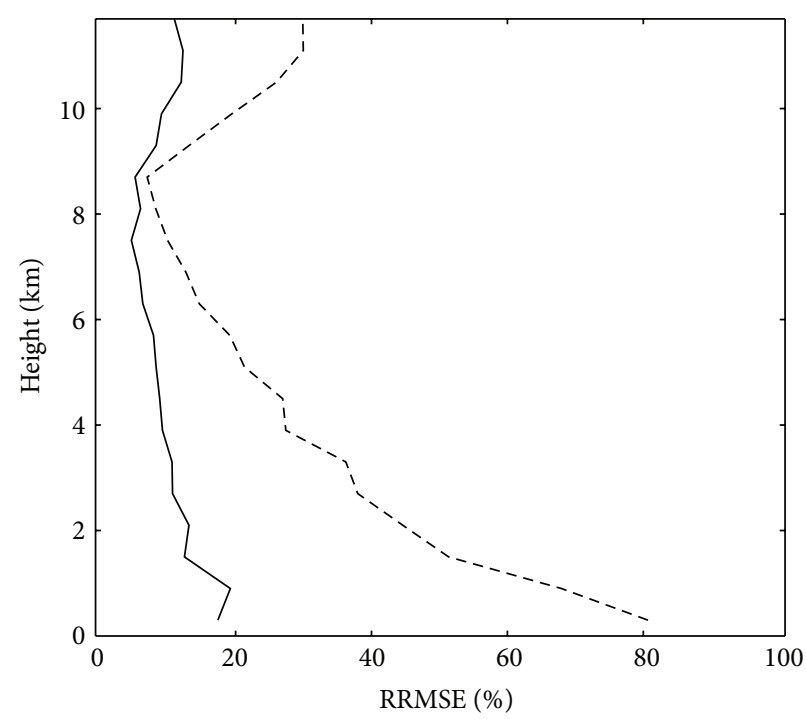

(b)

FIGURE 5: As in Figure 3 (top row) but for $p_{3 \mathrm{D}}$ (solid) and $p_{3 \mathrm{D}}$ retrieved with hydrometeor drag term omitted (dashed).

The final analysis $\pi_{\text {final }}$ is then obtained by subtracting the $A(k)$ estimate from $\pi_{2 D}$ :

$$
\pi_{\text {final }}=\pi_{2 \mathrm{D}}-\left\langle\pi_{2 \mathrm{D}}-\pi_{3 \mathrm{D}}\right\rangle .
$$

The $\pi_{\text {final }}$ is still subject to a volume-wide constant, which can be estimated as the volume-mean difference between $\pi_{\text {final }}$ and the $\pi$ from the simulation or EnKF analysis to which the retrieval method is applied. This estimate is reasonable to the extent that the errors in the input $\pi$ are unbiased. Errors in the estimate of this constant will not impact most applications since it is typically only the spatial derivatives of $\pi$ that are sought. The estimated volume-wide constant has been subtracted from the $\pi_{\text {final }}$ in the experiments below. To further facilitate interpretation, $\pi$ has been converted to dimensional perturbation pressure $p$.

\section{Experiments}

3.1. Verification of Retrieval Procedure. An NCOMMAS supercell simulation was used to test the robustness of the retrieval procedure and the integrity of the computer code. The simulation was performed on a stationary $102.6 \mathrm{~km} \times$ $102.6 \mathrm{~km} \times 20.4 \mathrm{~km}$ domain with $600 \mathrm{~m}$ grid spacing in all three dimensions and a large (small) time step of $4(2 / 3) \mathrm{s}$. A fully dual-moment version of the Ziegler [32] microphysical parameterization (Ziegler Variable Density or ZVD) scheme [33] was used. The remaining model settings were identical to those of Potvin et al. [23]. The supercell in the present simulation appears reasonably realistic, and its evolution qualitatively resembles that of Potvin et al. [23].

Pressure retrievals were performed over the entire simulation domain. The local derivatives of $u, v$, and $w$ were computed using centered finite differences with default
$\Delta t=30 \mathrm{~s}($ e.g., $\partial u / \partial t=(u(t+30)-u(t-30)) / 60)$. In general, spatial derivatives were computed using centered differencing on the Arakawa A (unstaggered) grid, necessitating averaging of the model $u, v$, and $w$ from the Arakawa C (staggered) grid. At the model boundaries, the $\pi$ derivatives in $J_{1}-J_{3}$ were computed using one-sided differences. In preliminary retrievals, setting the turbulence terms in the equations of motion to zero generally had negligible or mildly positive impact, presumably due to discretization errors being of similar magnitude to the turbulence terms themselves, which are typically much smaller than the remaining momentum equation terms. The turbulence terms were consequently omitted in the experiments shown.

Visual comparisons of the model and retrieved $p_{\text {final }}$, $\partial p_{\text {final }} / \partial x$, and $\partial p_{\text {final }} / \partial z$ reveal high fidelity in the retrieval technique (a representative example is shown in Figure 2), as do vertical profiles of root mean square error (RMSE) and relative (i.e., as a percentage of RMS $p_{\text {true }}$ ) RMSE (RRMSE) computed within the storm (Figure 3). (Both the model and retrieved pressure gradients were computed using centered differences on the Arakawa A grid.) Calculations of the horizontal domain-wide $p$ bias at each level indicate that the $3 \mathrm{D}$ retrieval largely eliminates the vertically varying constant present in the 2D retrieval (Figure 4). To assess the sensitivity of the technique to hydrometeor mixing ratio errors (which are often large in EnKF analyses due to gross imperfections in current microphysical parameterization schemes), the retrieval was repeated with the hydrometeor drag term (in the vertical equation of motion) omitted. While ignoring water loading substantially degraded $p_{3 \mathrm{D}}$ within the storm (Figure 5), $p_{3 \mathrm{D}}$ was barely impacted in precipitation-free regions (not shown), resulting in relatively small domain-wide $p_{3 \mathrm{D}}$ biases at each level and, thus, only minor error increases in the $A(k)$ estimates (Figure 4 ). The spurious vertical trend introduced to $p_{\text {final }}$ (generally < 

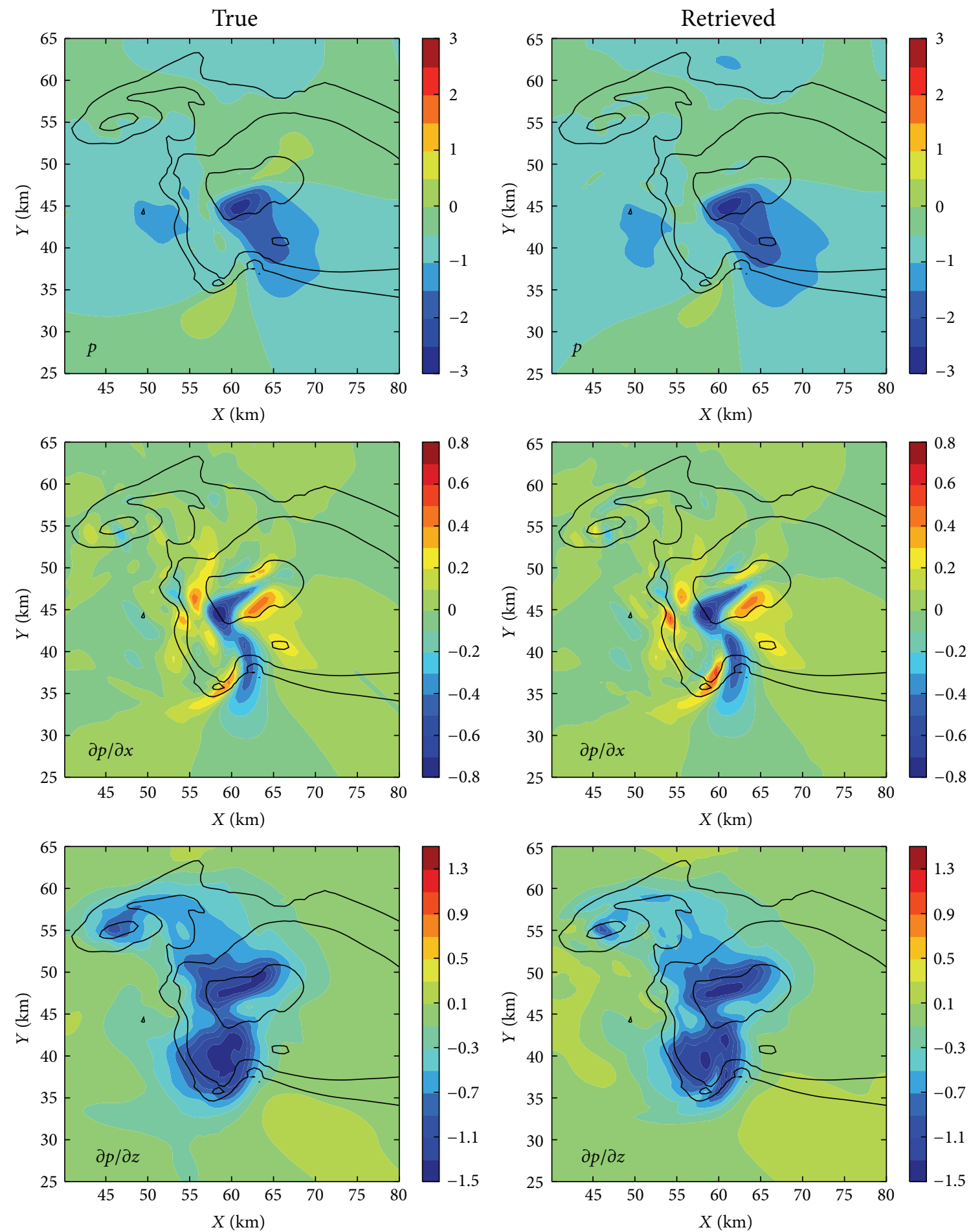

Figure 6: As in Figure 2 except for $\Delta t=120 \mathrm{~s}$ in the pressure retrieval.

$0.05 \mathrm{hPa} \mathrm{km}^{-1}$ ) by the degraded $A(k)$ was practically negligible. The relative insensitivity of the proposed retrieval method to hydrometeor errors is a major advantage over existing 3D approaches.

The retrievals were also relatively insensitive to temporal discretization errors in the local wind derivatives. Computing the latter using $\Delta t=120 \mathrm{~s}$ (rather than 30s) to match typical storm-scale EnKF analysis cycle periods substantially increased the RMSE $\partial p_{\text {final }} / \partial x$ and $\partial p_{\text {final }} / \partial z$ (Figure 3 ) but did not seriously qualitatively degrade the retrieval (Figure 6). While temporal discretization errors will be much larger in cases of rapidly moving storms (the present storm travels eastward at $\sim 10 \mathrm{~m} \mathrm{~s}^{-1}$ ), such errors may be substantially reduced using advection correction methods [34-36]. 

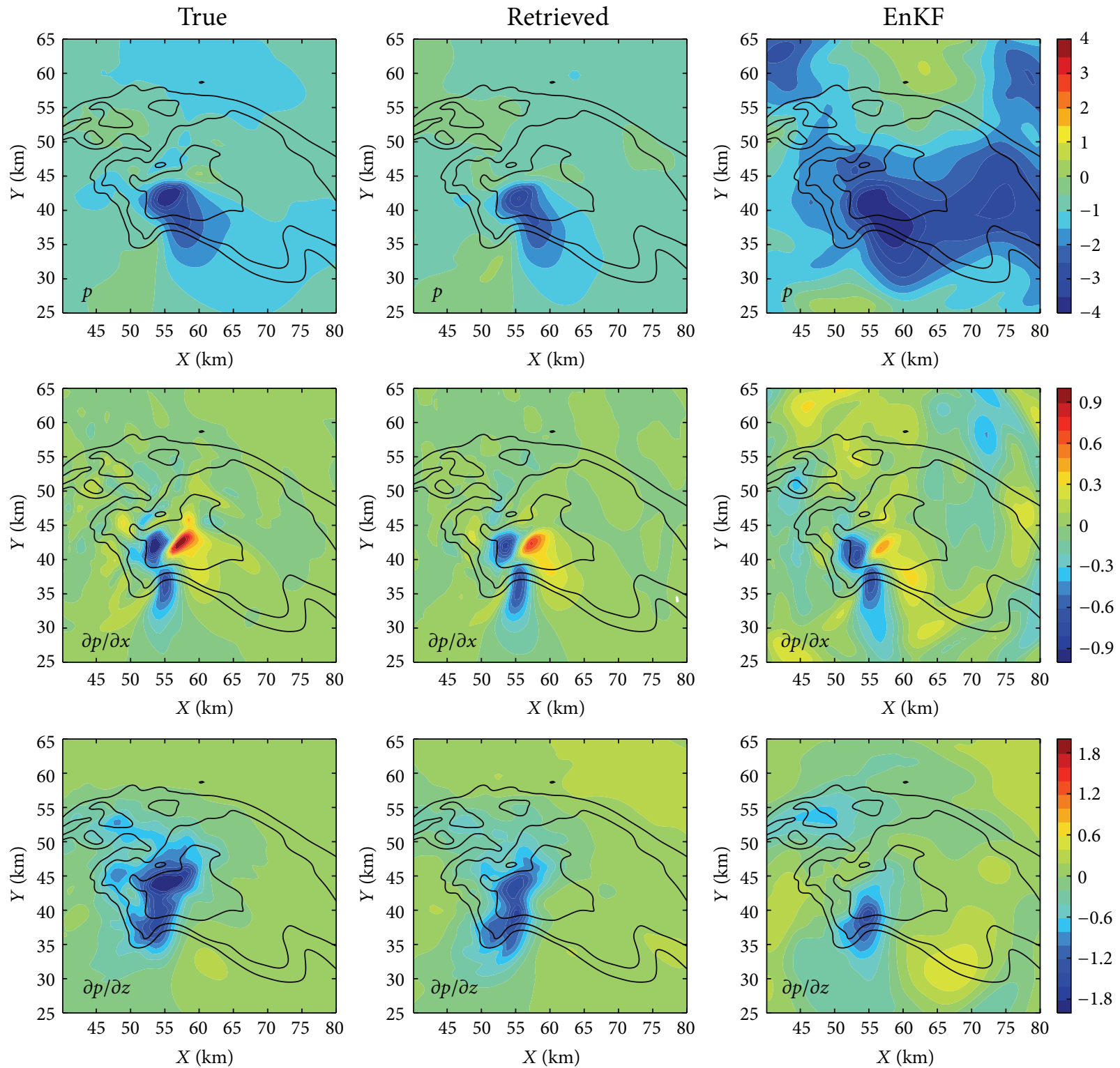

Figure 7: True (left column), retrieved (middle column), and EnKF mean posterior (right column) $p$ (top row), $\partial p / \partial x$ (middle row; $\mathrm{hPa} \mathrm{km}{ }^{-1}$ ), and $\partial p / \partial z$ (bottom row; $\mathrm{hPa} \mathrm{km}{ }^{-1}$ ) at $z=0.9 \mathrm{~km}, t=50 \mathrm{~min}$. The true $p$ were filtered and averaged as in Potvin et al. [23] to permit more direct comparisons with the (coarser) EnKF $p$. Radar reflectivity factor is contoured at 10, 30, and 50 dBZ.

3.2. Retrievals from a Simulated EnKF Mean Analysis. Having verified the robustness of the pressure retrieval scheme, we then applied the technique to mean posteriors from an EnKF OSSE conducted by Potvin and Wicker [11] (pictured in Figure 1). In that experiment (labeled "2-LFO" in [11]), mobile dual-Doppler velocity pseudoobservations were generated from the "truth" simulation of Potvin et al. [23], perturbed with random errors, and assimilated at two-minute intervals. Model error was introduced by using coarser ensemble grid spacing $(600 \mathrm{~m}$ versus $200 \mathrm{~m})$ and different microphysical parameterization (the Gilmore et al. [37] version of the Lin et al. [38] scheme versus the ZVD scheme) from those in the "truth" simulation. The use of an imperfect model in the EnKF resulted in dynamically unbalanced analysis increments that instigated acoustic waves during the data assimilation. The ensemble was integrated for $30 \mathrm{~min}$ prior to the first analysis update to develop physically realistic covariance structures.

Local wind derivatives for the pressure retrieval scheme were computed using mean posteriors separated by $4 \mathrm{~min}$ (i.e., $\Delta t=2 \mathrm{~min}$ ). This interval is similar to the volume scan periods for Weather Surveillance Radar-1988 Doppler (WSR-88D) convection sampling patterns. All fields required for the momentum equation constraints were obtained from the EnKF posteriors (i.e., treated as known quantities). The pressure retrievals were performed offline from the EnKF analysis; that is, the retrieved posterior pressure field at each time was not used to initialize the subsequent forecast cycle 

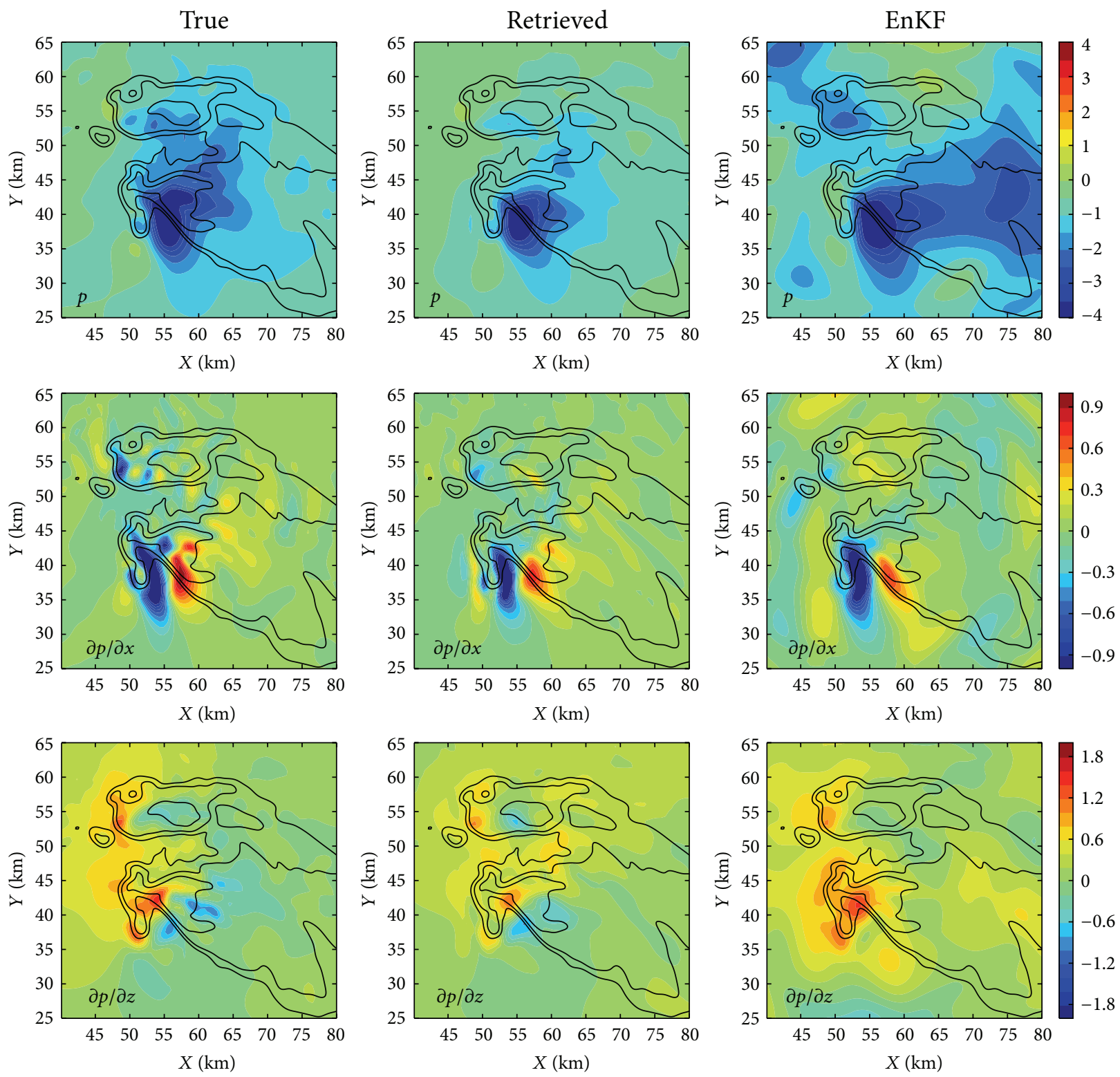

FIgURE 8: As in Figure 7 except for $z=3.3 \mathrm{~km}$.

in the data assimilation experiment. Due to the very weak impact of the pressure field on the remaining state variables in compressible models (the property exploited by the proposed method to remove the fast-mode pressure oscillations), rebalancing the pressure field at each analysis cycle should have generally negligible impact on the remaining model fields throughout the data assimilation. We have verified this with separate, real data experiments (not shown), in which the differences due to the pressure rebalancing produced only tiny differences in the final ensemble analysis and in subsequent $30 \mathrm{~min}$ forecasts.

Given the insensitivity of the pressure retrievals in Section 3.1 to discretization and hydrometeor mixing ratio errors, it was expected that $p_{\text {final }}$ obtained from the EnKF analyses would well capture the balanced portion of the
EnKF $p\left(p_{\text {bal }}\right)$ and thereby improve upon the EnKF $p$ and generally resemble $p_{\text {true }}$ (keeping in mind that errors in the other EnKF fields will create some discrepancies between the EnKF $p_{\text {bal }}$ and $\left.p_{\text {true }}\right)$. This was indeed the case throughout the assimilation period, except at higher altitudes. After $20 \mathrm{~min}$ of data assimilation $(t=50 \mathrm{~min})$, the EnKF wind analyses are sufficiently accurate that $p_{\text {final }}$ reasonably resembles $p_{\text {true }}$ at lower and middle levels, much more so than does the EnKF $p$ (Figures 7,8 , and 9). The improvement of $p_{\text {final }}$ over the EnKF p, however, decreases with height (Figure 9), presumably due in large part to the shallower fast-mode pressure oscillations aloft (not shown). Within the top half of the storm, $p_{\text {final }}$ is generally mildly inferior to the EnKF $p$, indicating that retrieval errors dominate improvements from removing the (small) fast-mode pressure errors. This 

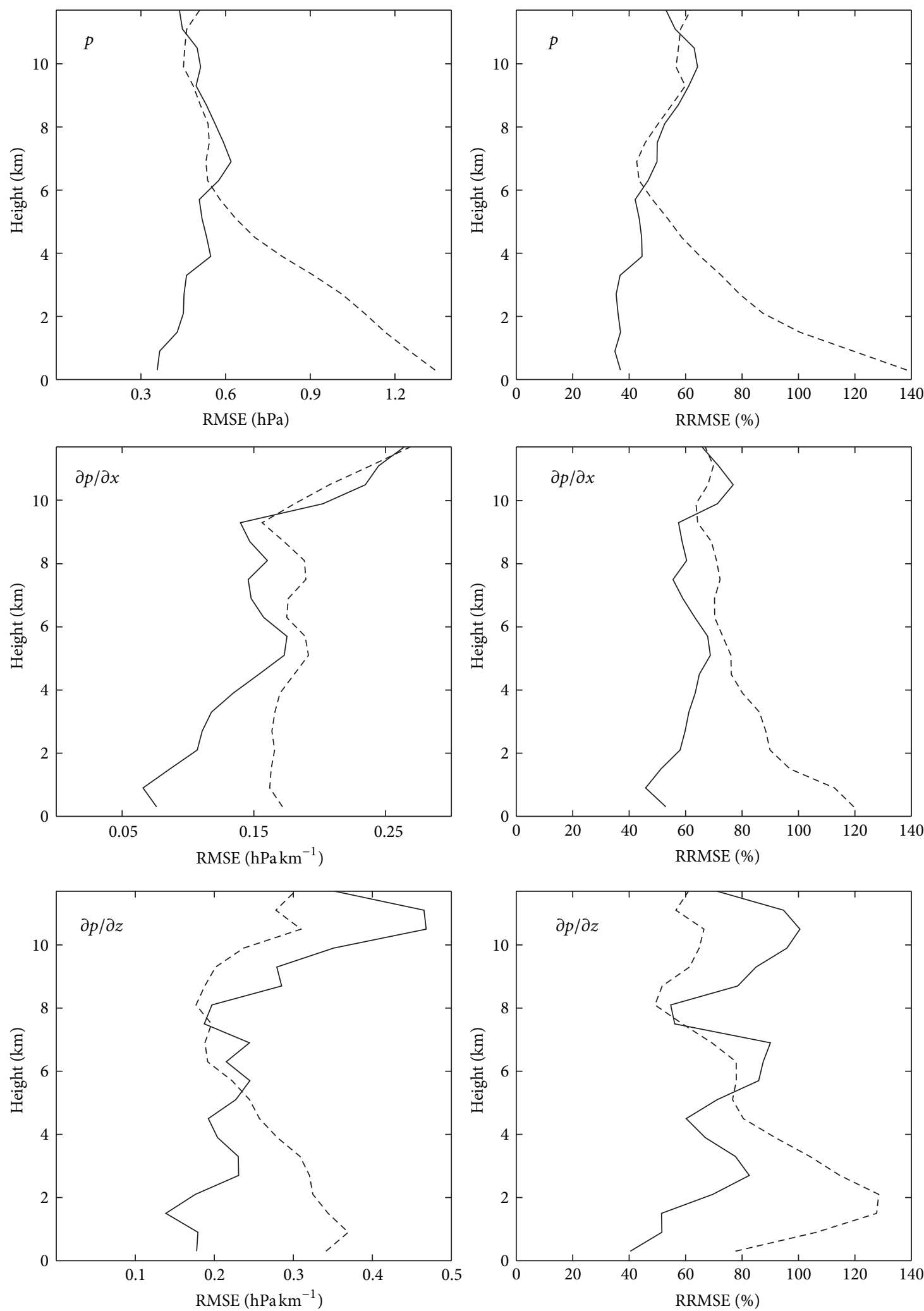

FIGURE 9: As in Figure 3 but for $p_{\text {final }}$ retrieved from mean EnKF posterior (solid) and mean EnKF posterior $p$ (dashed). 

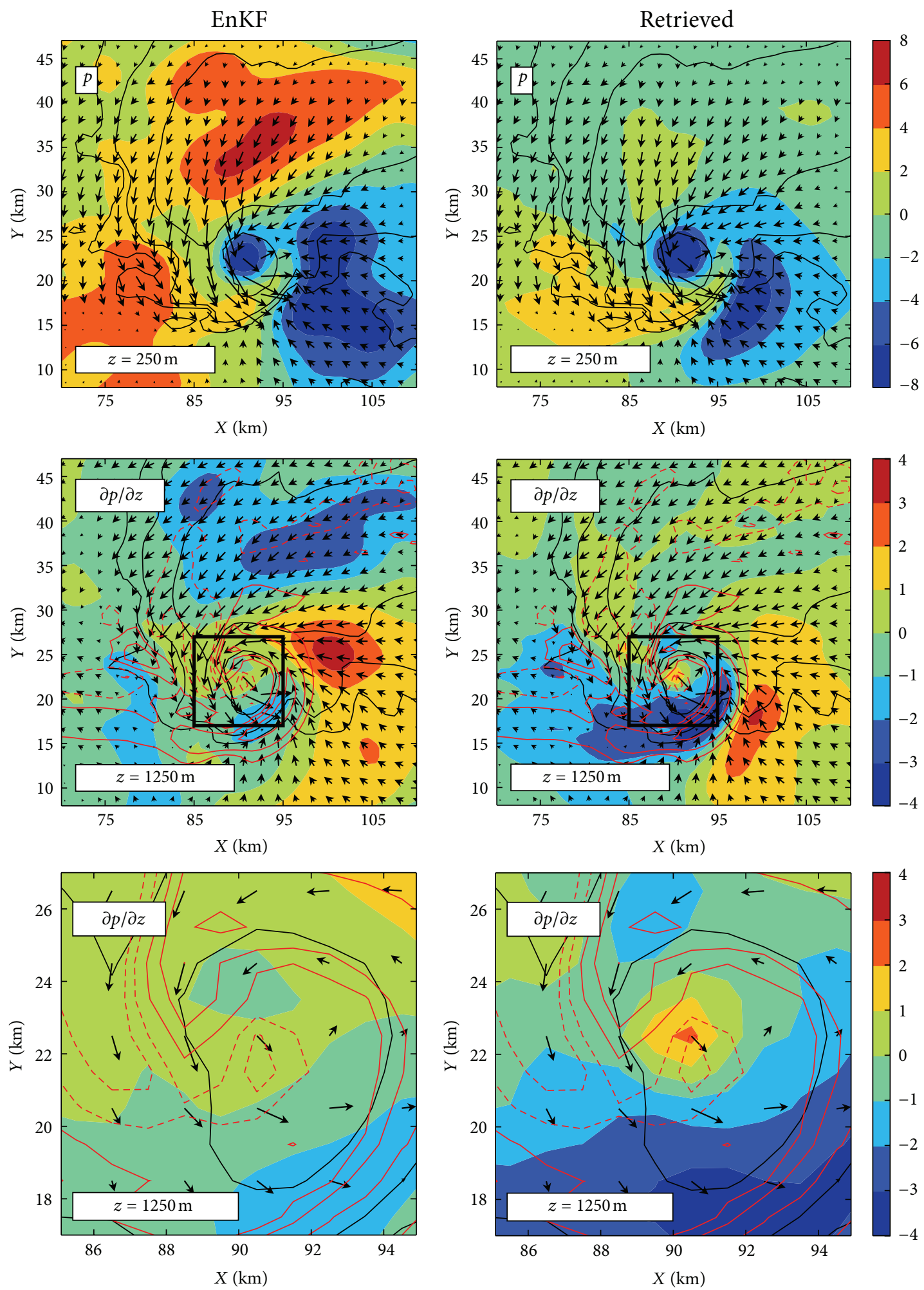

FIGURE 10: EnKF mean posterior (left column) and retrieved (right column) $p$ at $z=250 \mathrm{~m}$ (hPa; top row) and $\partial p / \partial z$ at $z=1250 \mathrm{~m}\left(\mathrm{hPa} \mathrm{km}{ }^{-1}\right.$; middle and bottom rows). The black squares in the middle row outline the domain (centered near the occlusion updraft) in the bottom row. EnKF mean posterior vertical velocity (red; dashed $=$ negative) is contoured at $-5,-2,2$, and $5 \mathrm{~m} \mathrm{~s}^{-1}$ and radar reflectivity factor (black) at 10, 30, and $50 \mathrm{dBZ}$. Arrows represent EnKF mean posterior horizontal winds. 
suggests that application of the proposed pressure retrieval method be restricted to altitudes where spurious pressure oscillations are large.

While the retrieval results from this OSSE are likely somewhat optimistic despite our efforts to mitigate the "identical twin" problem, larger EnKF analysis errors in real cases should not substantially impede the technique's ability to recover the EnKF $p_{\text {bal }}$ and, thus, to remove the fastmode pressure errors (which is the objective of the proposed method). Furthermore, the previous results, combined with the resilience of dual-mobile-Doppler EnKF wind analyses to microphysical and background wind errors [11, 39], suggest that pressure retrievals obtained from high-quality radar datasets such as those collected during the second Verification of the Origins of Rotation in Tornadoes Experiment (VORTEX2; [40]) may be useful for investigating pressuregradient-driven storm processes. Rigorous testing of this hypothesis, unfortunately, is currently precluded by the lack of dense $3 \mathrm{D}$ pressure observations within storms.

3.3. Retrievals from a Real Data EnKF Mean Analysis. While our ability to verify the real-world performance of the proposed technique is quite limited, qualitative evaluation of pressure retrievals from EnKF analyses of the May 29-30, 2004, Geary, OK, USA, tornadic supercell supports cautious optimism. The EnKF analyses were obtained by assimilating quality-controlled Doppler velocity data from two Shared Mobile Atmospheric Research and Teaching (SMART; [41]) radars using the NCOMMAS EnKF with the ZVD microphysics scheme (see Potvin et al. [39] for further details). Pressure retrievals were performed in the same manner as with the simulated EnKF analyses in Section 3.2.

A representative comparison of the $p_{\text {final }}$ and EnKF $p$, valid after 44 min of data assimilation (0037 UTC 30 May), is shown in Figure 10. As in the EnKF OSSE (Section 3.2), highamplitude pressure oscillations generated during the data assimilation are not evident in the retrieved pressure fields. Furthermore, the $p_{\text {final }}$ comports with present understanding of the pressure distribution within supercells, which suggests retrieval errors are not unduly large. For example, both the $p_{\text {final }}$ and EnKF $p$ exhibit an inflow low and a deep pressure minimum associated with the strong mesocyclonic rotation (Figures 10(a) and 10(b)), as well as upward-directed perturbation pressure gradient force along the gust front (Figures $10(\mathrm{c})$ and $10(\mathrm{~d})$ ). The $p_{\text {final }}$, but not the EnKF $p$, persistently indicates a region of substantial downward-directed perturbation pressure gradient force near the analyzed occlusion downdraft (Figures 10(c)-10(f)). This is consistent with current understanding of occlusion downdraft formation [42], suggesting that the EnKF pressure analysis near the occlusion downdraft is contaminated with fast-mode errors that the retrieval successfully corrects.

\section{Conclusion}

As in other data assimilation frameworks, storm-scale EnKF radar data assimilation can introduce dynamical imbalances that induce severe pressure errors in analyses, inhibiting investigation of important storm processes. Retrieval procedures that have traditionally been used to retrieve the pressure and buoyancy fields from dual-Doppler wind analyses can be used to rebalance the pressure field in such cases. Using a combination of the $2 \mathrm{D}$ and $3 \mathrm{D}$ pressure retrieval approaches that mitigates the deficiencies of both, we have demonstrated that useful analyses of the perturbation pressure field and its derivatives can be obtained from EnKF analyses despite model and discretization errors. The described procedure could be modified to separately retrieve, for example, the linear and nonlinear components of the dynamic perturbation pressure [43]. The method could also be extended to other nonhydrostatic equation sets including that used by the WRF-ARW model.

It should be noted that some of the existing methods for suppressing acoustic and gravity waves in model initial conditions could be adapted to removing pressure oscillations in EnKF analyses. For example, during data assimilation, digital filter initialization (DFI; [44]) could be applied to the analysis increments at each cycle prior to integrating the ensemble forward to the next time. All such approaches, however, would increase computational cost, and it is not clear whether storm-scale analyses would improve given the model approximations used in these methods (e.g., the adiabatic backward model integration in DFI). Alternatively, a diagnostic pressure equation (e.g., [45]) could be solved (either exactly, or weakly as in the proposed method), thereby avoiding temporal discretization errors, but at the cost of increased spatial discretization errors. While we recommend evaluation of the relative efficacy of alternative methods for recovering the balanced pressure component, we also emphasize that the proposed method provides an existing, effective, simple, and low-cost way to remove pressure oscillations from storm-scale EnKF analyses and does not require data assimilation experiments to be rerun.

\section{Acknowledgments}

Funding was provided by the NOAA/Office of Oceanic and Atmospheric Research under NOAA-University of Oklahoma Cooperative Agreement no. NA11OAR4320072, US Department of Commerce. This work benefitted from discussions with Patrick Skinner and Alan Shapiro. The authors thank the three anonymous reviewers for their helpful comments that substantially improved the paper and Michael French for his useful comments on an earlier version of the paper. The SMART radar data were collected and provided by Michael Biggerstaff and edited by Kristin Kuhlman and Daniel Betten under NSF Grants ATM-0619715 and ATM-0802717.

\section{References}

[1] G. Evensen, "Sequential data assimilation with a nonlinear quasi-geostrophic model using Monte Carlo methods to forecast error statistics," Journal of Geophysical Research, vol. 99, no. 5, pp. 10-162, 1994. 
[2] C. Snyder and F. Q. Zhang, "Assimilation of simulated Doppler radar observations with an ensemble Kalman filter," Monthly Weather Review, vol. 131, no. 8, pp. 1663-1677, 2003.

[3] F. Q. Zhang, C. Snyder, and J. Z. Sun, "Impacts of initial estimate and observation availability on convective-scale data assimilation with an ensemble Kalman filter," Monthly Weather Review, vol. 132, no. 5, pp. 1238-1253, 2004.

[4] D. C. Dowell, F. Zhang, L. J. Wicker, C. Snyder, and N. A. Crook, "Wind and temperature retrievals in the 17 May 1981 Arcadia, Oklahoma, supercell: ensemble Kalman filter experiments," Monthly Weather Review, vol. 132, no. 8, pp. 1982-2005, 2004.

[5] M. J. Tong and M. Xue, "Ensemble Kalman filter assimilation of Doppler radar data with a compressible nonhydrostatic model: OSS experiments," Monthly Weather Review, vol. 133, no. 7, pp. 1789-1807, 2005.

[6] M. Xue, M. J. Tong, and K. K. Droegemeier, "An OSSE framework based on the ensemble square root Kalman filter for evaluating the impact of data from radar networks on thunderstorm analysis and forecasting," Journal of Atmospheric and Oceanic Technology, vol. 23, no. 1, pp. 46-66, 2006.

[7] A. Aksoy, D. C. Dowell, and C. Snyder, "A multicase comparative assessment of the ensemble Kalman filter for assimilation of radar observations. Part I: storm-scale analyses," Monthly Weather Review, vol. 137, no. 6, pp. 1805-1824, 2009.

[8] D. T. Dawson, L. J. Wicker, E. R. Mansell, and R. L. Tanamachi, "Impact of the environmental low-level wind profile on ensemble forecasts of the 4 may 2007 Greensburg, Kansas, tornadic storm and associated mesocyclones," Monthly Weather Review, vol. 140, no. 2, pp. 696-716, 2012.

[9] D. C. Dowell, L. J. Wicker, and C. Snyder, "Ensemble kalman filter assimilation of radar observations of the 8 may 2003 oklahoma city supercell: influences of reflectivity observations on storm-scale analyses," Monthly Weather Review, vol. 139, no. 1, pp. 272-294, 2011.

[10] J. Marquis, Y. Richardson, P. Markowski, D. Dowell, and J. Wurman, "Tornado maintenance investigated with high-resolution dual-doppler and EnKF analysis," Monthly Weather Review, vol. 140, no. 1, pp. 3-27, 2012.

[11] C. K. Potvin and L. J. Wicker, "Comparison between DualDoppler and EnKF Storm-Scale Wind Analyses: observing system simulation experiments with a supercell thunderstorm," Monthly Weather Review, vol. 140, no. 12, pp. 3972-3991, 2012.

[12] R. L. Tanamachi, L. J. Wicker, D. C. Dowell, H. B. Bluestein, D. T. Dawson, M. Xue et al., "EnKF assimilation of highresolution, mobile Doppler radar data of the 4 May 2007 Greensburg, Kansas, Supercell into a numerical cloud model," Monthly Weather Review, vol. 141, no. 2, pp. 625-648, 2013.

[13] L. J. Wicker and W. C. Skamarock, "Time-splitting methods for elastic models using forward time schemes," Monthly Weather Review, vol. 130, no. 8, pp. 2088-2097, 2002.

[14] M. C. Coniglio, D. J. Stensrud, and L. J. Wicker, "Effects of upper-level shear on the structure and maintenance of strong quasi-linear mesoscale convective systems," Journal of the Atmospheric Sciences, vol. 63, no. 4, pp. 1231-1252, 2006.

[15] J. S. Whitaker and T. M. Hamill, "Ensemble data assimilation without perturbed observations," Monthly Weather Review, vol. 130, no. 7, pp. 1913-1924, 2002.

[16] J. Anderson, T. Hoar, K. Raeder et al., "The data assimilation research testbed a community facility," Bulletin of the American Meteorological Society, vol. 90, no. 9, pp. 1283-1296, 2009.
[17] W. C. Skamarock, J. B. Klemp, J. Dudhia et al., "A description of the Advanced Research WRF version 2," Tech. Rep., NCAR, 2005.

[18] J. B. Klemp and R. Rotunno, "A study of the tornadic region within a supercell thunderstorm," Journal of the Atmospheric Sciences, vol. 40, no. 2, pp. 359-377, 1983.

[19] J. D. Marwitz, "Trajectories within the weak echo region of hailstorms," Journal of Applied Meteorology, vol. 12, no. 7, pp. 1174-1182, 1973.

[20] R. Rotunno and J. B. Klemp, “The influence of shear-induced pressure gradient on thunderstorm motion," Monthly Weather Review, vol. 110, no. 2, pp. 136-151, 1982.

[21] R. A. Houze Jr., S. A. Rutledge, M. I. Biggerstaff, and B. F. Smull, "Interpretation of Doppler weather radar displays of midlatitude mesoscale convective systems," Bulletin of the American Meteorological Society, vol. 70, no. 6, pp. 608-619, 1989.

[22] Z. Byko, P. Markowski, Y. Richardson, J. Wurman, and E. Adlerman, "Descending reflectivity cores in supercell thunderstorms observed by mobile radars and in a high-resolution numerical simulation," Weather and Forecasting, vol. 24, no. 1, pp. 155-186, 2009.

[23] C. K. Potvin, L. J. Wicker, and A. Shapiro, "Assessing errors in variational dual-doppler wind syntheses of supercell thunderstorms observed by storm-scale mobile radars," Journal of Atmospheric and Oceanic Technology, vol. 29, no. 8, pp. 10091025, 2012.

[24] R. Daley, Atmospheric Data Analysis, Cambridge Atmospheric and Space Science Series, Cambridge University Press, Cambridge, UK, 1991.

[25] D. T. Kleist, D. F. Parrish, J. C. Derber, R. Treadon, R. M. Errico, and R. Yang, "Improving incremental balance in the GSI 3DVAR analysis system," Monthly Weather Review, vol. 137, no. 3, pp. 1046-1060, 2009.

[26] T. Galchen, "Method for initialization of anelastic equationsimplications for matching models with observations," Monthly Weather Review, vol. 106, no. 5, pp. 587-606, 1978.

[27] C. E. Hane, R. B. Wilhelmson, and T. Gal-Chen, "Retrieval of thermodynamic variables within deep convective clouds: experiments in three dimensions," Monthly Weather Review, vol. 109, no. 3, pp. 564-576, 1981.

[28] E. A. Brandes, "Relationships between radar-derived thermodynamic variables and tornadogenesis," Monthly Weather Review, vol. 112, no. 5, pp. 1033-1052, 1984.

[29] F. Roux, "Retrieval of thermodynamic fields from multipleDoppler radar data using the equations of motion and the thermodynamic equation," Monthly Weather Review, vol. 113, no. 12, pp. 2142-2157, 1985.

[30] Y. C. Liou, T. C. C. Wang, and K. S. Chung, "A threedimensional variational approach for deriving the thermodynamic structure using Doppler wind observations-an application to a subtropical squall line," Journal of Applied Meteorology, vol. 42, no. 10, pp. 1443-1454, 2003.

[31] J. B. Klemp and R. B. Wilhelmson, "Simulation of 3-dimensional convective storm dynamics," Journal of the Atmospheric Sciences, vol. 35, no. 6, pp. 1070-1096, 1978.

[32] C. L. Ziegler, "Retrieval of thermal and microphysical variables in observed convective storms. Part 1: model development and preliminary testing," Journal of the Atmospheric Sciences, vol. 42, no. 14, pp. 1487-1509, 1985. 
[33] E. R. Mansell, C. L. Ziegler, and E. C. Bruning, "Simulated electrification of a small thunderstorm with two-moment bulk microphysics," Journal of the Atmospheric Sciences, vol. 67, no. 1, pp. 171-194, 2010.

[34] T. G.-C. Tzvi Gal-Chen, "Errors in fixed and moving frame of references: applications for conventional and Doppler radar analysis," Journal of the Atmospheric Sciences, vol. 39, no. 10, pp. 2279-2300, 1982.

[35] A. Shapiro, K. M. Willinghamm, and C. K. Potvin, "Spatially variable advection correction of radar data. Part I: theoretical considerations," Journal of the Atmospheric Sciences, vol. 67, no. 11, pp. 3445-3456, 2010.

[36] A. Shapiro, K. M. Willingham, and C. K. Potvin, "Spatially variable advection correction of radar data. Part II: test results," Journal of the Atmospheric Sciences, vol. 67, no. 11, pp. 3457-3470, 2010.

[37] M. S. Gilmore, J. M. Straka, and E. N. Rasmussen, "Precipitation uncertainty due to variations in precipitation particle parameters within a simple microphysics scheme," Monthly Weather Review, vol. 132, no. 11, pp. 2610-2627, 2004.

[38] Y.-L. Lin, R. D. Farley, and H. D. Orville, "Bulk parameterization of the snow field in a cloud model," Journal of Climate \& Applied Meteorology, vol. 22, no. 6, pp. 1065-1092, 1983.

[39] C. K. Potvin, J. W. Louis, M. I. Biggerstaff, D. Betten, and A. Shapiro, "Comparison between Dual-Doppler and EnKF storm-scale wind analyses: the 29-30 May 2004 Geary, Oklahoma, Supercell thunderstorm," Monthly Weather Review, vol. 141, no. 5, pp. 1612-1628, 2013.

[40] J. Wurman, D. Dowell, Y. Richardson et al., "The second verification of the origins of rotation in tornadoes experiment Vortex2," Bulletin of the American Meteorological Society, vol. 93, no. 8, pp. 1147-1170, 2012.

[41] M. I. Biggerstaff, L. J. Wicker, J. Guynes et al., "The shared mobile atmospheric research and teaching radar: a collaboration to enhance research and teaching," Bulletin of the American Meteorological Society, vol. 86, no. 9, pp. 1263-1274, 2005.

[42] P. M. Markowski, "Hook echoes and rear-flank downdrafts: a review," Monthly Weather Review, vol. 130, no. 4, pp. 852-876, 2002.

[43] H. Cai and R. M. Wakimoto, "Retrieved pressure field and its influence on the propagation of a supercell thunderstorm," Monthly Weather Review, vol. 129, no. 11, pp. 2695-2713, 2001.

[44] P. Lynch and X.-Y. H. Xiang-Yu Huang, "Initialization of the HIRLAM model using a digital filter," Monthly Weather Review, vol. 120, no. 6, pp. 1019-1034, 1992.

[45] G. Q. Ge, J. D. Gao, and M. Xue, "Diagnostic pressure equation as a weak constraint in a storm-scale three-dimensional variational radar data assimilation system," Journal of Atmospheric and Oceanic Technology, vol. 29, no. 8, pp. 1075-1092, 2012. 

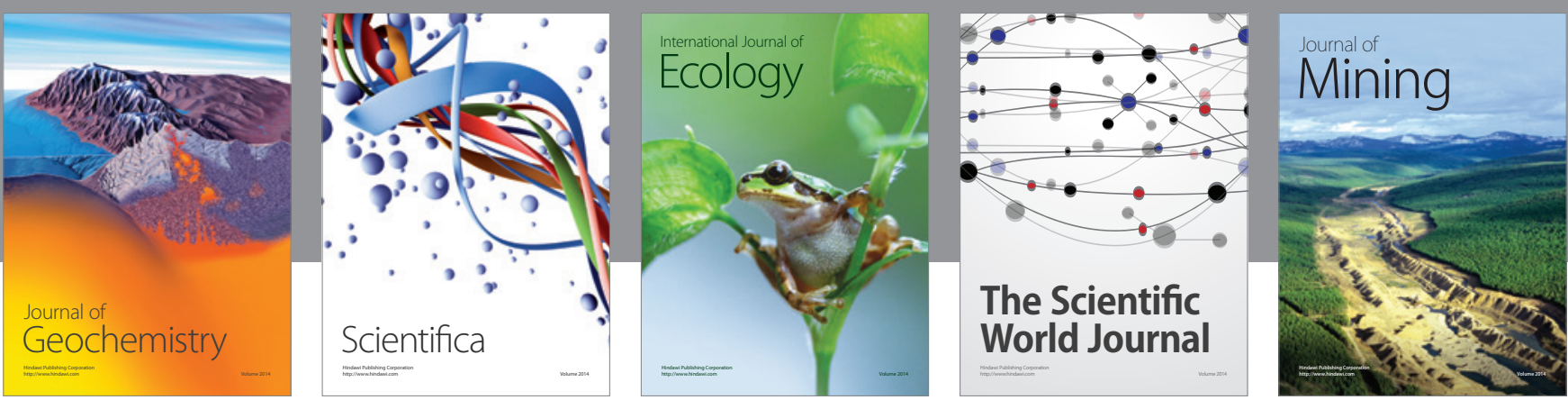

The Scientific World Journal
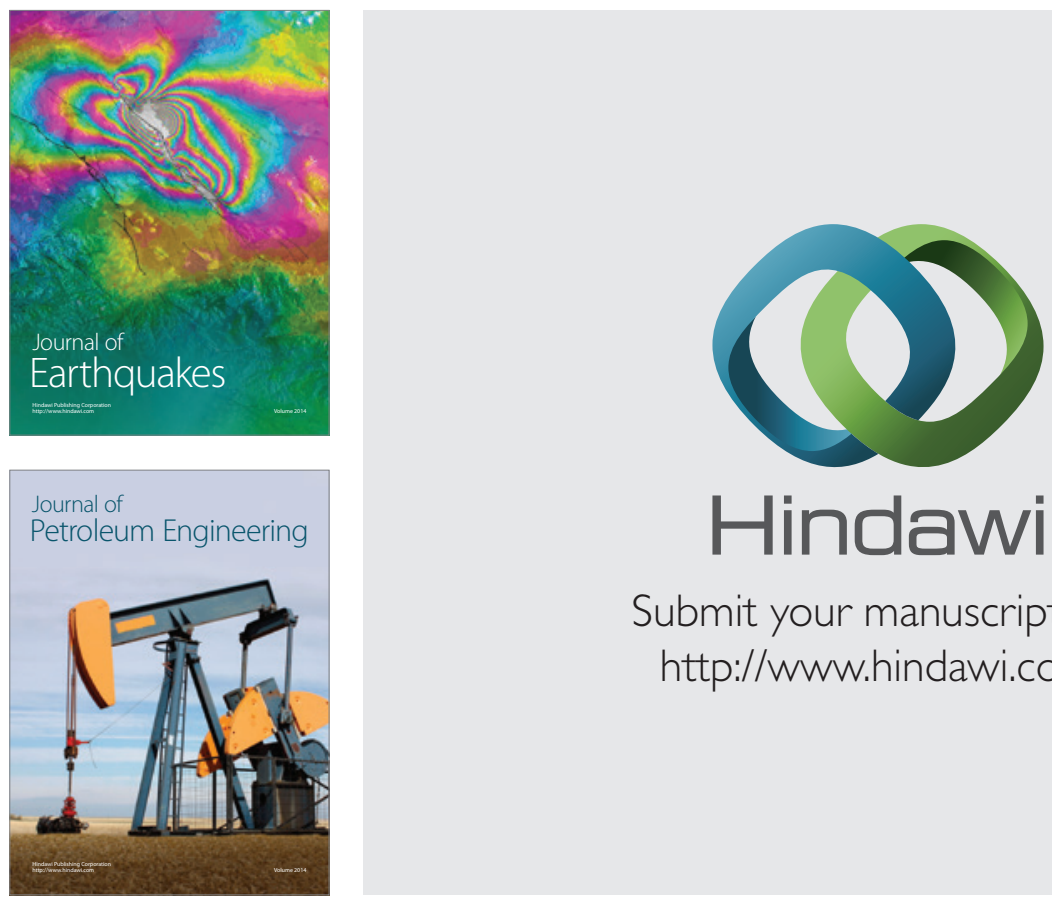

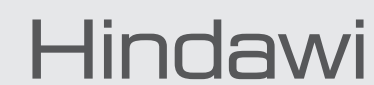

Submit your manuscripts at

http://www.hindawi.com
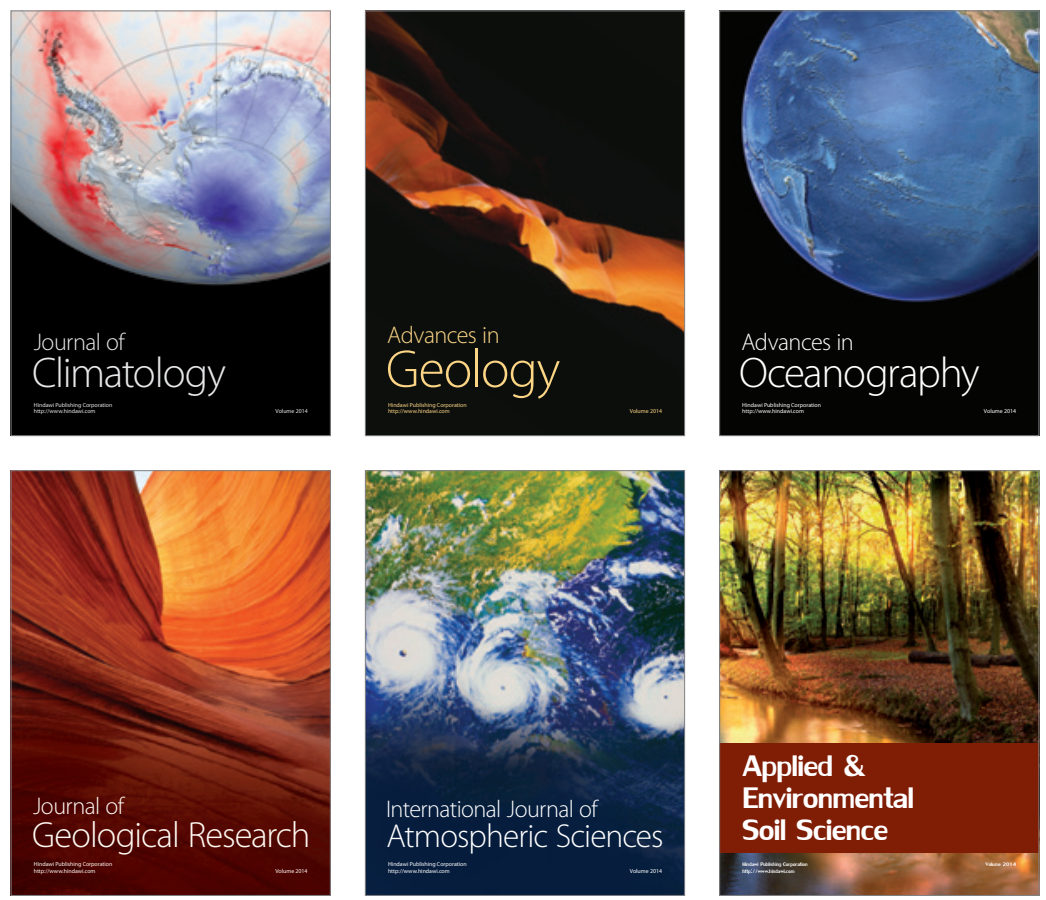
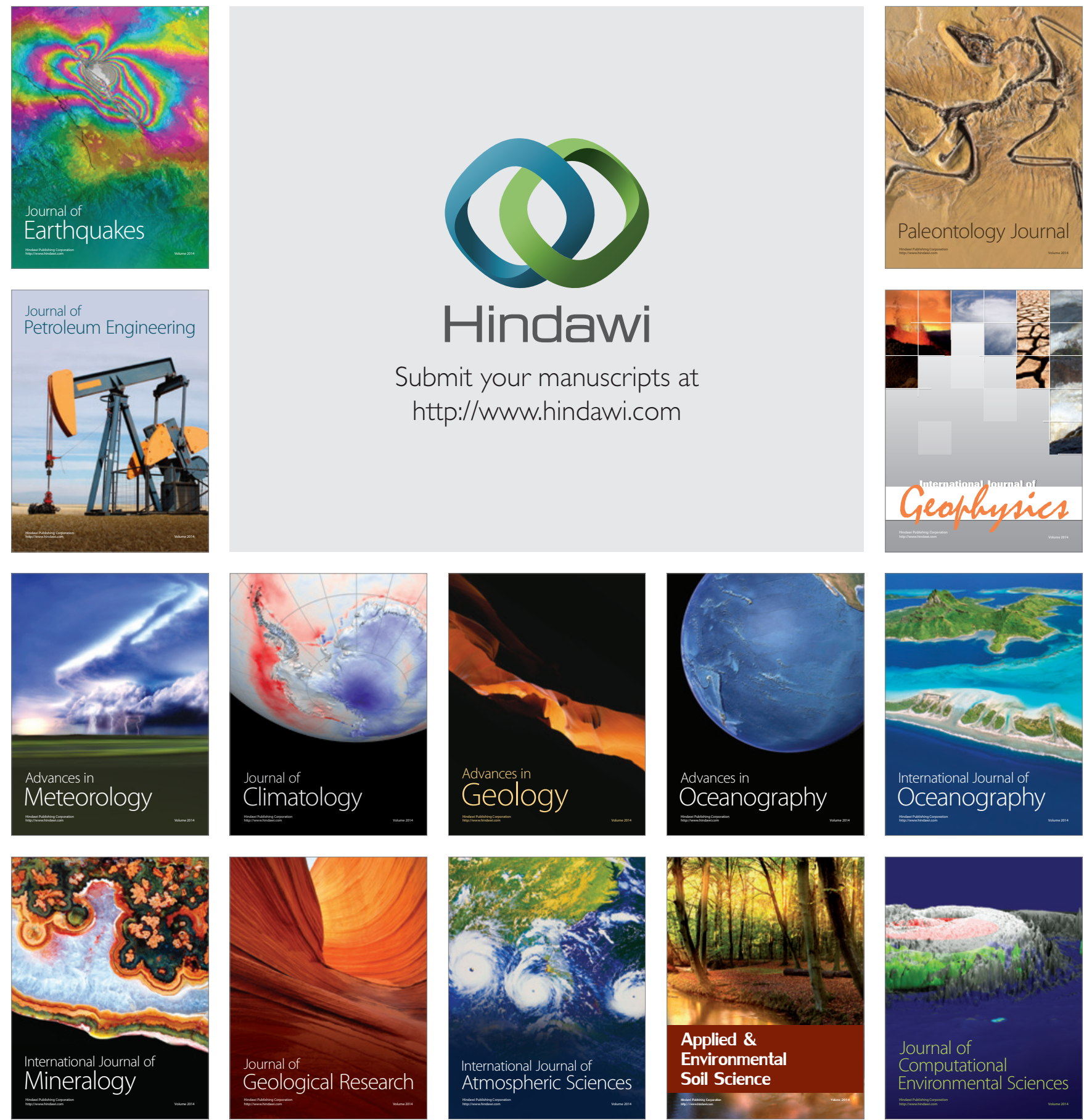